\title{
A Study on Motion Control of Multiple Floating Units
}

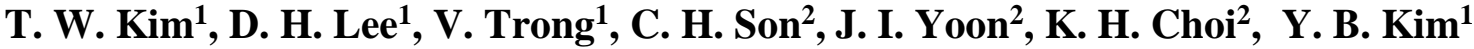 \\ ${ }^{1}$ Department of Mechanical System Engineering, Pukyong National University \\ Nam-gu, Busan, Korea \\ kpjiwoo@pknu.ac.kr \\ ${ }^{2}$ Department of Refrigeration and Air-conditioning Engineering, Pukyong National University \\ Nam-gu, Busan, Korea
}

\begin{abstract}
This article describes the Pontoon Bridge installation problem. The Pontoon Bridge is called as Foldable Bridge which was designed to carry a heavy vehicles and trucks as a floating bridge or ferry by connecting several or many number of floating units. Until now, the installation job is almost done by hand without considering the worst cases which illustrate the disaster and hard environmental situation. It means that the installation job may be impossible in the hailed bullets situation. In this paper, the authors propose a new installation strategy to overcome all situations including the wartime.
\end{abstract}

Keywords: Pontoon Bridge, Heavy Vehicle, Floating Bridge, Disaster, Installation.

\section{Introduction}

Temporal bridging for river-crossing is a great important operation for emergency restoration in both military and civil field. In term of bridging, the pontoon bridge [1] is constructed by connecting many floating units on rivers or seas. Two major factors that decide the success of the pontoon bridge construction are speedy and safety. Conventionally, the installation process and linearity maintaining of the pontoon bridge are carried out by employing the erection boats that operated by number of operators as shown in Fig.1. It may be a solution to overcome the difficulties by using winch system and active bridge in which power propulsion system is installed (shown in Fig. 2 [2]).

However, it is confirmed that maintaining the placement of every single floating unit under variety of affections including water flow current, moving load, waves is difficult. Therefore, this operation is considered as one of the most dangerous operation, especially in combat situation. To ensure the speedy and safety of the river-crossing operation, the automated installation and displacement correction should be applied.

In recent years, a certain number of researchers paid attention to the pontoon floating bridge. S. Fu and W. Cui presented the dynamic analysis of floating bridges [3]. Besides, Hirono et al. [4] introduced a new study on measurement system for positional displacement of floating units for pontoon bridges. Most recently, Nguyen et al. [5] presented the mathematical modelling and numerical investigation of the pontoon ribbon bridge such that it enables the possibility of automated installation and yaw-displacement correction. Where, the power propulsion system is installed and controlled by the designed control system.

To comprehensively investigate the issue about installation and operation of the automated multi-connected floating unit bridge systems, we propose an applicable control method for ensuring the linearity among connected bays. And based on that, the bridge installation is archived by the yaw motion control. In this study, we develop an optimal controller with a state estimator based on mathematical model to simulate the three-connected-bay system model installation and its operation with disturbances. To verify the efficiency of the designed controller, a number of numerical simulations are investigated with commercial software Matlab and Simulink.

To further validate the proposed control strategy and numerical simulation results, the experimental studies are carried out. The numerical and experiment study results demonstrate that our designed controller and state estimator are eligible for the pontoon bridge installation and operation automatically. 


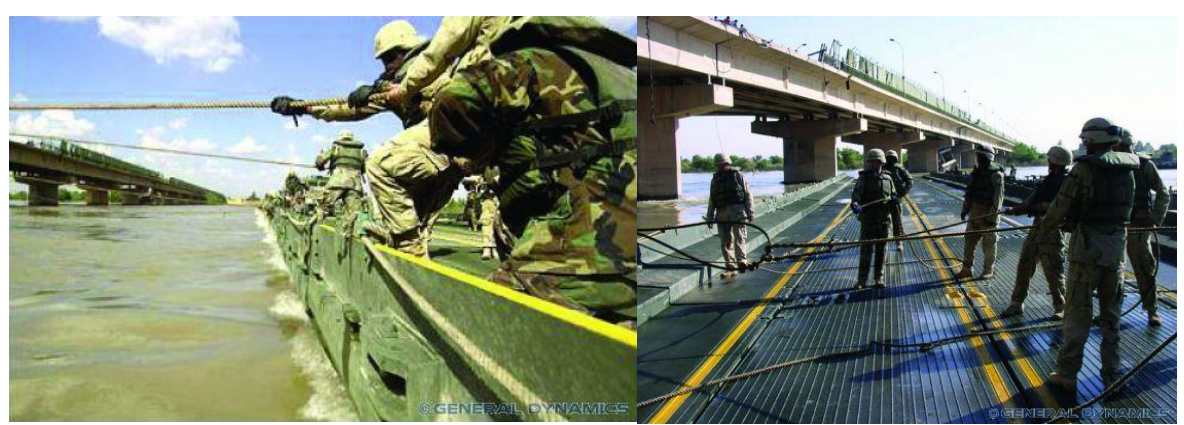

Fig. 1: An example of ribbon bridge installation process operated by human power [1].

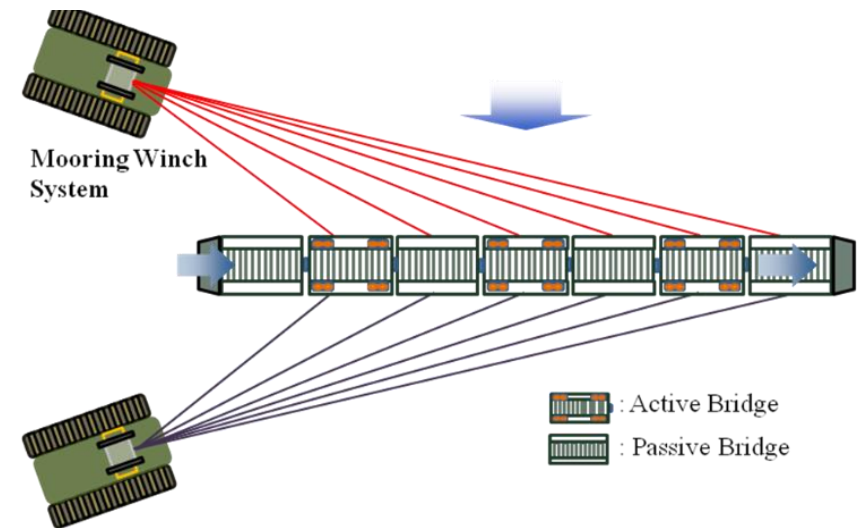

Fig. 2: An installation strategy (cascade type installation method) [2].

\section{Modelling and Controller Design}

\subsection{System Description}

The basis motion modelling of the floating units consists of the kinematic and kinetic model and is represented as follows:

$$
J \dot{v}+C_{v} v=\tau
$$

Where, $\tau$ is the control input composed forces and moments produced by propulsion systems, $v=[u, v, r]^{T} \in R^{3}$ describes the surge, sway, and yaw rate of the floating unit motion in body fixed coordinate frame, $J \in R^{3 \times 3}$ presents a mass/inertia matrix, $C_{v}$ is the hydrodynamic damping matrix:

$$
J=\left[\begin{array}{ccc}
m-X_{\dot{u}} & 0 & 0 \\
0 & m-Y_{\dot{v}} & 0 \\
0 & 0 & I_{z}-N_{\dot{r}}
\end{array}\right]
$$

and

$$
C_{v}=\left[\begin{array}{ccc}
-X_{u} & 0 & 0 \\
0 & -Y_{v} & 0 \\
0 & 0 & -N_{r}
\end{array}\right]
$$


Where $\mathrm{m}$ is the mass, and $I_{z}$ is the inertia moment of the floating unit on the fixed z-axis, respectively. Generally, the planar motions of this multi-connected floating units shown in Fig. 3 are described as following:

$$
J_{i} \ddot{\theta}_{i}+C_{v} \dot{\theta}_{i}+C_{i-1}\left(\dot{\theta}_{i}-\dot{\theta}_{i-1}\right)+C_{i}\left(\dot{\theta}_{i}-\dot{\theta}_{i+1}\right)=\left(F_{i-1}^{l u}+F_{i-1}^{l d}+F_{i}^{r u}+F_{i}^{r d}\right) h+\left(F_{i}^{p}-F_{i}^{w}\right) w_{v}
$$

where $i=0,1, \ldots, n$. Based on this fact, in this paper we consider a control system design problem for controlling three-bay pontoon bridge in a designed manner. In details, the mathematical model of three-bay pontoon bridge can be written as follows:

$$
\begin{gathered}
J_{1} \ddot{\theta}_{1}+C_{v} \dot{\theta}_{1}+C_{0} \dot{\theta}_{1}+C_{1}\left(\dot{\theta}_{1}-\dot{\theta}_{2}\right) \\
=\left(F_{0}^{l u}+F_{0}^{l d}+F_{1}^{r u}+F_{1}^{r d}\right) h+\left(F_{1}^{p}-F_{1}^{w}\right) w_{v} \\
J_{2} \ddot{\theta}_{2}+C_{v} \dot{\theta}_{2}+C_{1}\left(\dot{\theta}_{2}-\dot{\theta}_{1}\right)+C_{2}\left(\dot{\theta}_{2}-\dot{\theta}_{3}\right) \\
=\left(F_{2}^{r u}+F_{2}^{r d}+F_{1}^{l u}+F_{1}^{l d}\right) h+\left(-F_{2}^{w}\right) w_{v} \\
J_{3} \ddot{\theta}_{3}+C_{v} \dot{\theta}_{3}+C_{2}\left(\dot{\theta}_{3}-\dot{\theta}_{2}\right)+C_{3} \dot{\theta}_{3} \\
=\left(F_{2}^{l u}+F_{2}^{l d}\right) h+\left(F_{3}^{p}-F_{3}^{w}\right) w_{v}
\end{gathered}
$$

In (5) (7), $C_{i}, \theta_{i}(i=0,1,2,3)$ are the damping coefficient and the yaw rate of the corresponding floating unit, respectively; $F_{i}^{l u}, F_{i}^{l d}$ are the spring forces at the left hand-side of the vessel; similarly, $F_{i}^{r u}, F_{i}^{r d}$ are the spring forces at the right hand-side of the vessel. $F_{j}^{P}(j=1,2)$ are the driven forces generated by the propulsion systems. Because the propulsion systems are installed in the first and third floating unit from the left in Fig. 2, respectively. $F_{k}^{w}(k=1,2,3)$ are the water current flow forces. $h$ and $w_{v}$ are the corresponding arm forces for spring forces and driven forces, respectively.
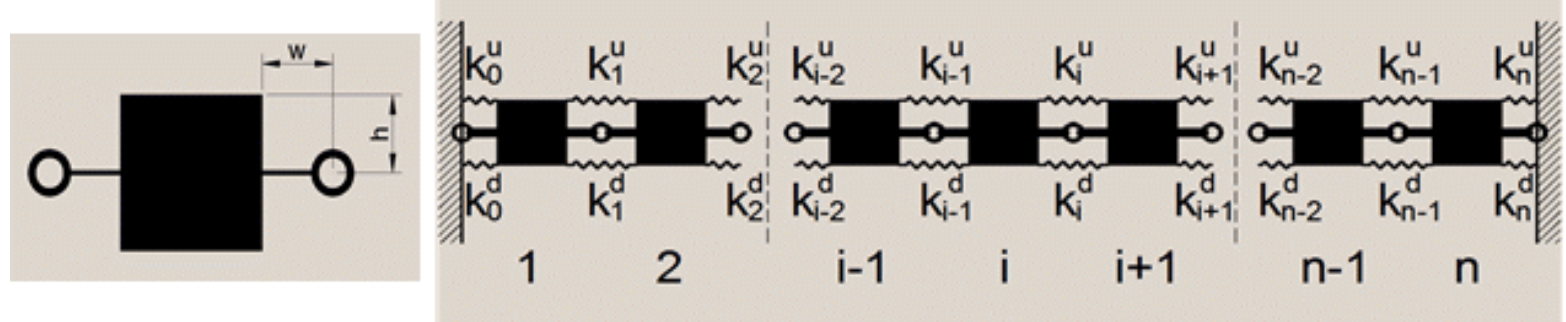

Fig. 3: The schematic diagram of three-floating unit bridge system for experiment.

\subsection{Controller Design with Observer}

The aim of the control system design is controlling the motions of floating units and keeping the multiple connected pontoon bridge system in the defined position. As abovementioned, in this paper, the controlled system is made by three floating units with two propulsion systems installed on first and third unit. It means that there is no propulsion apparatus in the second floating unit. We can find out this fact from equation (6). However, let us illustrate a servosystem with observer (shown in Fig. 4) to estimate states and obtain specified control performance. 


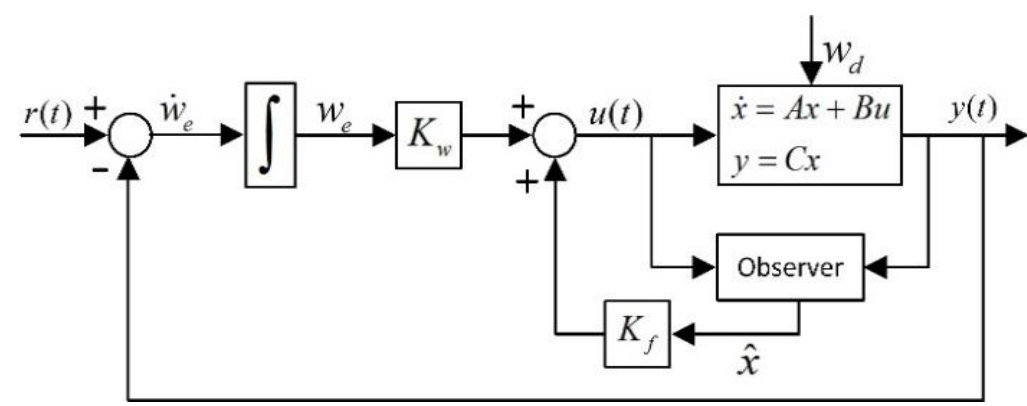

Fig. 4: A servosystem with observer for controlling floating bridge position.

The parameter values appeared in Eqs. (5) (7) are obtained by several experiments and evaluated by numerical calculations. Using the identified system model, the controller gains including observer gain L appeared in Fig. 4 are obtained and applied to real experiment system. The results are shown in the next section.

\section{Simulation and Experiment}

The In this section, experiment results are illustrated by using the designed control system with gains obtained by optimal control theory. For this, the authors set up the experimental apparatus as shown in Fig. 5. Based on the simulation study, several experimental studies have been executed to evaluate the performance and robustness of the proposed control system. In our system, there are two active floating units with integrated propulsion systems which can produce adjusting forces and one passive floating unit inserted between two active ones. It means that the motor-driven propulsion systems are installed in first and third floating unit (illustrated as active bays in Fig. 5). And the yaw motions of all floating units are measured by three incremental encoders.

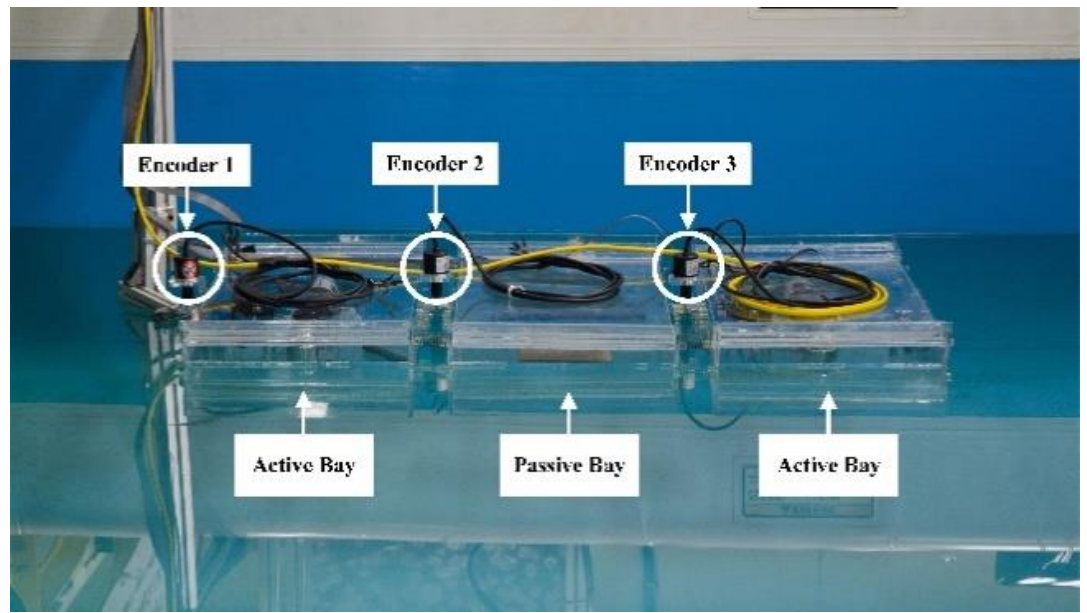

Fig. 5: The experiment setup for floating bridge system.

The experiment result describes the yaw motion of the bridge system with the $0^{\circ}$ initial point and $90^{\circ}$ final target point. It is a kind of step response. The all floating units should be kept in parallel with $0^{\circ}$ relative angle in the steady state. Fig. 6 shows the absolute yaw deviation of each floating unit in calm water (no wave disturbance). The target position is archived smoothly with a minor overshoot then remaining at the desired point. It confirms that our proposed control strategy for bridge installation with observer-based has remarkable possibility for multi-connected pontoon bridge control. 


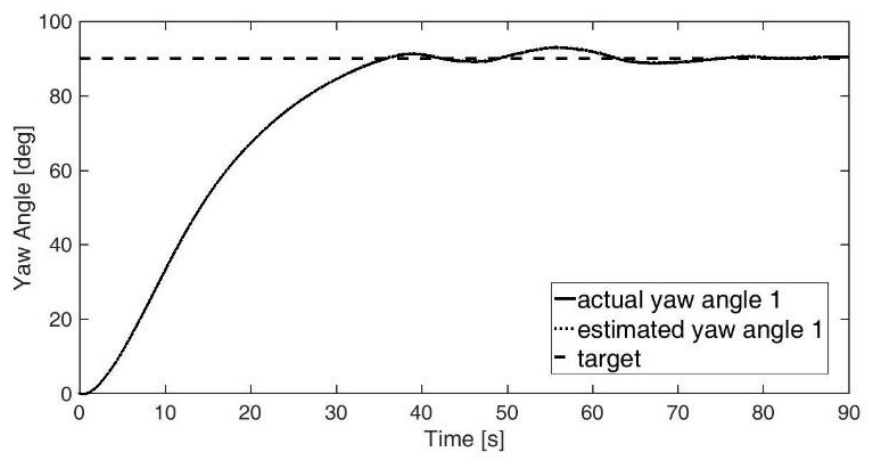

(a) yaw angle of \#1 unit

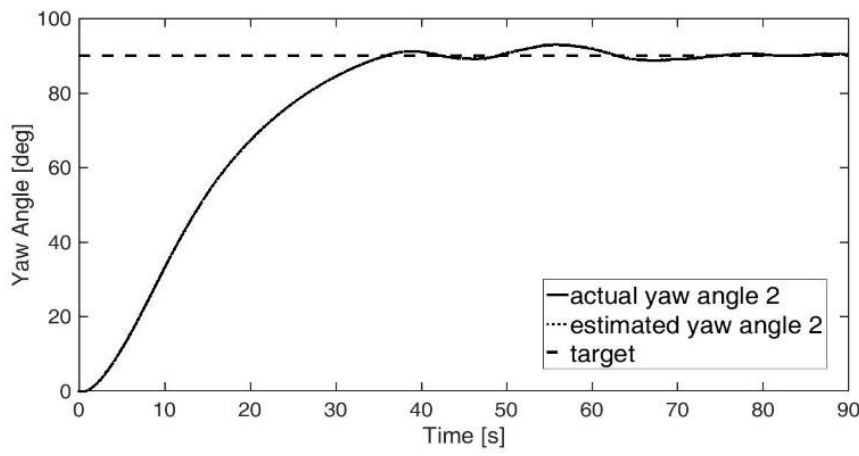

(b) yaw angle of \#2 unit

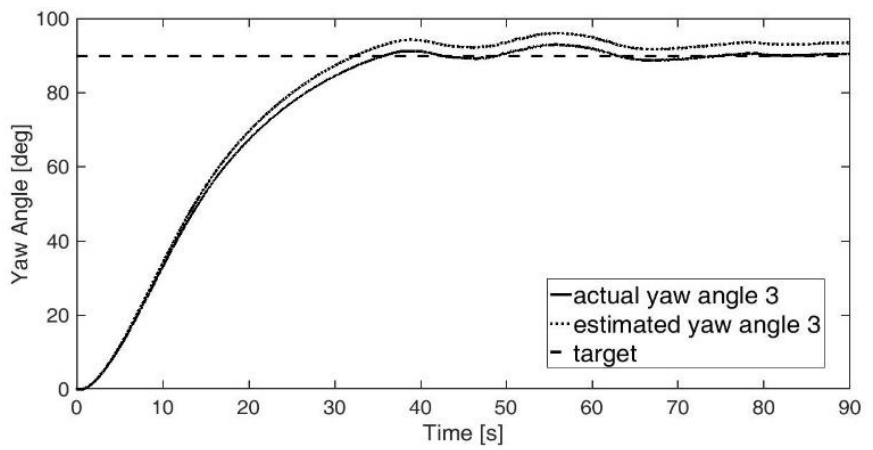

(c) yaw angle of \#3 unit

Fig. 6: Controlled yaw motions in calm water (experiment result).

\section{Conclusion}

In this study, we have proposed a control strategy for installing the floating bridge system constructed by several floating units. For the control system design, a servosystem with estimator is introduced to take unmeasurable states such that and the linearity of the bridge system is to be kept. The simulation and experimental results give something valuable facts that the promising feasibility of positional linearity control and automated installation of floating bridge system without any human power can be realized.

\section{Acknowledgements}

This work was supported by the National Research Foundation of Korea (NRF) grant funded by the Korea Government (Ministry of Education) (No.NRF-2015R1D1A1A09056885).

\section{References}

[1] General Dynamics, Improved Ribbon Bridge, 2012.

[2] Y. Kim, "Pontoon bridge installation method," Korean Patent, 10 1627827, 2016. 
[3] S. Fu and W. Cui, "Dynamic response of a ribbon floating bridge under moving loads," Marine Structures, vol. 11, pp. 29-46, 1998.

[4] Y. Hirono, Y. Mizuchi, Y. Kim and Y. Choi, "Positional displacement measurement of floating units based on aerial images for pontoon bridges," Lecture Notes on Electrical Engineering, Springer, vol. 415, pp. 309-318, 2016.

[5] V. T. Nguyen, M. S. Choi and Y. Kim, "A study on automated ribbon bridge installation strategy," Journal of Institute of Control, Robotics and Systems, vol. 23, no. 8, pp. 661-666, 2017. 